REVUE DE L'INSTITUT

FRANÇAIS D'HISTOIRE

EN ALLEMAGNE

\section{Revue de l'IFHA}

Revue de l'Institut français d'histoire en Allemagne

$2 \mid 2010$

IFHA 2

\title{
Élaborer, écrire et diffuser l'histoire de la « Grande Révolution française » dans les social-démocraties allemande et autrichienne 1889-1934
}

Jean-Numa Ducange

\section{OpenEdition}

\section{Journals}

Édition électronique

URL : http://journals.openedition.org/ifha/252

DOI : $10.4000 /$ ifha. 252

ISSN : 2198-8943

Éditeur

IFRA - Institut franco-allemand (sciences historiques et sociales)

\section{Édition imprimée}

Date de publication : 1 janvier 2010

Pagination : 201-208

ISSN : 2190-0078

\section{Référence électronique}

Jean-Numa Ducange, «Élaborer, écrire et diffuser l'histoire de la « Grande Révolution française » dans les social-démocraties allemande et autrichienne 1889-1934 », Revue de l'IFHA [En ligne], 2 | 2010, mis en ligne le 01 février 2013, consulté le 03 mai 2019. URL : http://journals.openedition.org/ifha/252 ; DOI : $10.4000 /$ ifha.252

Ce document a été généré automatiquement le 3 mai 2019.

(C)IFHA 


\section{Élaborer, écrire et diffuser l'histoire de la « Grande Révolution française » dans les social- démocraties allemande et autrichienne 1889-1934}

Jean-Numa Ducange

\section{NOTE DE L'ÉDITEUR}

Thèse soutenue le 30 novembre 2009 à l'Université de Rouen devant un jury comprenant : M. Michel Biard (Université de Rouen, président du jury), M. Paul Pasteur (Université de Rouen, directeur de recherche), M. Michael Werner (EHESS, rapporteur), M. Serge Wolikow (Université de Bourgogne, rapporteur), M. Matthias Middell (Université de Leipzig)

Notre thèse de doctorat s'inscrit dans le prolongement de recherches menées dans le cadre d'un mémoire de maîtrise puis d'un master sur les écrits du social-démocrate Karl Kautsky traitant de la Révolution française, en particulier son ouvrage sur les contradictions de classes en 1789 traduit en français en $1901^{1}$. Celui-ci parut en France au moment où Jean Jaurès commence à publier son Histoire socialiste de la Révolution française, présentée souvent par l'historiographie comme une œuvre initiatrice d'une longue tradition d'étude. Si quelques travaux avaient souligné le contenu et la réception de ces deux œuvres dans leurs espaces nationaux respectifs, aucun n'avait restitué jusqu'ici la confrontation croisée de leurs points de vue. Cette recherche nous a conduit à constater l'importance de la production des sociaux-démocrates allemands et autrichiens sur la "Grande Révolution française » à l'échelle d'un demi-siècle. Alors même que l'historiographie de la Révolution française connaît de nouveau un regain d'intérêt ${ }^{2}$ et 
que de nouvelles approches de l'histoire sociale des mouvements ouvriers ${ }^{3}$ et de la production intellectuelle qui lui est liée se font jour ${ }^{4}$, trois interrogations sont rapidement apparues.

\section{Historiographie, « marxisme », histoire croisée}

2 En premier lieu, nous nous sommes interrogé sur le lien entre production historique et débat politique, sur les usages multiples de l'histoire de la Révolution dans le cadre des partis sociaux-démocrates. En parallèle d'ouvrages sur la période 1789-1799 paraissent en effet nombre d'articles interrogeant le ou les modèle(s) politique(s) à partir de l'expérience révolutionnaire passée. Plusieurs thèmes, qui ont animé par la suite des décennies d'historiographie, connaissent à la fin $d u$ dix-neuvième siècle des développements singuliers ; parmi eux, citons la nature du processus révolutionnaire (en particulier l'attention portée à des forces sociales comme la bourgeoisie), la place d'un épisode controversé comme celui de la Terreur (et les problèmes qu'elle pose en rapport avec la politique sociale-démocrate), ou encore la genèse d'un récit qui fait de certains épisodes voire de certains révolutionnaires français des précurseurs de l'idée socialiste (la place du babouvisme est à cet égard décisive).

3 Deuxièmement, constatant que Kautsky fut le premier à présenter une histoire systématique de la Révolution française inspirée par le marxisme, comment définir et présenter l'historiographie "marxiste», terme aux déclinaisons multiples? Nous nous sommes inspiré de la définition proposée par Georges Haupt, historien décédé prématurément en 1978, ayant quitté la Roumanie à la fin des années 1950 pour la France. Devenu directeur d'études à l'EHESS, il impulsa une série de travaux sur l'histoire des mouvements ouvriers internationaux. Souhaitant rompre avec les histoires produites par les appareils politiques, Georges Haupt incitait à étudier le «marxisme » en historicisant le terme. Il prit pour base les premiers textes s'y référant dans les années 1880, soit une définition à partir des usages concrets dans les textes de la social-démocratie allemande et plus largement dans l'espace de la Seconde Internationale ${ }^{5}$.

Enfin, notre recherche devait se situer hors d'une stricte histoire nationale, dans des problématiques issues des «transferts culturels » qui ont inspiré nombre de travaux sur les rapports entre la France et les pays germaniques ${ }^{6}$. Mais plus que la seule réception de la Révolution française en Allemagne et en Autriche, la compréhension de la construction d'une lecture spécifique de la Révolution française doit aussi s'envisager au regard des débats avec le socialisme français. Les outils méthodologiques proposés par l'« histoire croisée » ont servi à analyser ces rapports complexes au-delà des seuls effets de transferts et des comparaisons classiques entre les divers espaces nationaux ${ }^{7}$. Plusieurs travaux inscrits dans ce sillage sur la question de la traduction ont été mobilisés; l'enjeu important du choix de traduire certains ouvrages et de ne pas en traduire d'autres, afin de comprendre par exemple l'introduction des problématiques jaurésiennes, a constitué un de nos fils conducteurs ${ }^{8}$.

\section{Production intellectuelle et pratiques militantes}

5 La façon dont se construit une lecture singulière de la Révolution française doit s'envisager au travers d'ouvrages, brochures, articles et comptes rendus écrits par les 
sociaux-démocrates allemands et autrichiens sans oublier les publications que ceux-ci ont traduites et introduites. Dans cette optique, certaines figures comme Karl Kautsky, pour le lien qu'il représente entre l'Autriche et l'Allemagne mais aussi en raison de ses rapports avec les socialistes français, Heinrich Cunow (en particulier pour son ouvrage consacré à la presse révolutionnaire ${ }^{9}$ ) ou encore Hermann Wendel connu pour sa biographie de Danton ${ }^{10}$, ont été étudiées avec attention. Ce retour aux textes de l'époque nous a permis de retrouver des contributions jamais reparues depuis, par exemple des articles de Franz Mehring et Rosa Luxemburg sur la Terreur de $1793^{11}$.

6 Ce premier ensemble - dont l'objectif était de restituer l'histoire de la production intellectuelle des sociaux-démocrates sur la Révolution française - s'est progressivement combiné avec une histoire plus organisationnelle se démarquant des approches susceptibles de différencier, voire d'opposer l'histoire politique à l'histoire intellectuelle. À distance des approches traditionnelles et dans un souci de comprendre les usages des références historiques dans les organisations politiques, nous nous sommes appuyé sur l'historiographie récente des pratiques militantes, sensible à l'abondante littérature produite par les organisations sociales-démocrates ${ }^{12}$. Des travaux sur d'autres forces politiques étudiant la construction de la vulgate guesdiste en France, l'histoire des communismes ou encore "l'invention des traditions», ont été aussi mobilisés ${ }^{13}$. Ainsi, si ce sont avant tout les livres, brochures, et articles des revues théoriques comme Die Neue Zeit et Der Kampf traitant de la "Grande Révolution» qui ont retenu notre attention, d'autres sources, habituellement peu consultées et mises en avant par ces nouvelles recherches, ont servi d'appui à notre étude. Répartis dans différents centres d'archives, principalement à l'IISG (Internationaal Instituut voor Sociale Geschiedenis - Institut International d'Histoire Sociale) d'Amsterdam et la Friedrich-Ebert-Stiftung de Bonn ${ }^{14}$, les almanachs ouvriers (Arbeiter-Kalender), journaux commémoratifs, journaux quotidiens, les livres empruntés dans les bibliothèques du parti comme les revues de formation destinées aux cadres constituent un vaste ensemble permettant de cerner la constitution et la diffusion d'une vulgate sur l'histoire de la Révolution française. Le domaine de la formation, la Bildung (qui peut être traduit par éducation, formation, culture), concept d'origine libérale réinvesti par la social-démocratie pour ses entreprises militantes, a fait l'objet d'une attention particulière. Le fonctionnement des écoles de formation et quelques figures intermédiaires comme les Wanderlehrer - professeurs itinérants - ont été présentés au travers d'exemples régionaux permettant d'esquisser une histoire de la transmission d'une référence historique dans ces partis à différents niveaux. Les plans détaillés des formations ont constitué une source importante, ceux-ci étant souvent, comme nombre d'articles, des reprises simplifiées d'ouvrages plus élaborés. Ainsi peut-on mesurer l'impact de la vulgate, la façon dont celle-ci se constitue : un ouvrage historique sur la Révolution française, parfois peu lu, pouvait avoir un écho bien plus large si l'on tient compte de toutes les déclinaisons dont il était l'objet.

\section{Chronologies croisées}

7 Cet ensemble de publications a sa chronologie propre, parfois liée à des commémorations ponctuelles comme le centenaire de 1889 à 1894. Chronologie qui rencontre les événements marquant l'histoire des social-démocraties; l'articulation entre les deux permet de suivre les usages successifs de la référence à la Révolution française. Sans exclusive, la légalisation du SPD et la mise en place du dispositif de formation au début 
des années 1890, la crise révisionniste et notamment les positions respectives d'Eduard Bernstein et de Jean Jaurès, les révolutions russes de 1905 et 1917, la Première Guerre mondiale, les révolutions allemande et autrichienne de 1918-1919, enfin les conflits politiques de l'Allemagne de Weimar et de l'Autriche de la Première République, particulièrement dans ce dernier cas les années qui mènent à la guerre civile de février 1934, sont l'occasion de relire la séquence de 1789-1799, souvent pour confronter par analogies les différentes voies offertes par un processus révolutionnaire ${ }^{15}$. Si les chronologies sont diverses et s'il n'existe pas de lien mécanique entre un événement et une lecture définie de l'histoire de la Révolution française, les résonances entre un nouveau processus révolutionnaire, l'élaboration théorique et la diffusion de la vulgate ne sont pas à négliger. Pour ne citer qu'un exemple, la révolution de 1905 stimule des analogies - un 1789 russe a-t-il lieu? - (élaboration théorique), contribue à de nombreuses publications et rééditions (écriture de l'histoire) et remet à l'honneur dans de nombreux supports la diffusion d'une vulgate (par exemple le retour de la période 1789-1799 dans les plans de formation d'une école du parti). Le poids de la conjoncture politique est aussi essentiel pour comprendre par exemple la publication en Autriche de l'ouvrage d'Emma Adler sur les femmes pendant la Révolution française : elle constitue une réaction à l'acceptation du suffrage universel masculin en 1905 mettant en sourdine les revendications des femmes ${ }^{16}$.

8 Les ruptures d'après-guerre, en particulier la Révolution russe de 1917, stimulent de nombreuses analogies tandis que les recompositions politiques qu'elle entraîne, notamment le ralliement des partis sociaux-démocrates à l'État, sont à l'origine de nouvelles lectures qui ne peuvent se comprendre sans les profonds changements des conditions de la production intellectuelle. Parmi eux, citons les liens avec les universités : Hedwig Hintze, Privat-Dozentin proche du SPD introduit Alphonse Aulard et Albert Mathiez dans un environnement globalement hostile aux traditions révolutionnaires. Dans le même temps, les écrits publiés avant 1914 connaissent toujours une diffusion, mais dans des cadres diversifiés : les communistes du KPD, souvent d'anciens membres du SPD, fondent leur matérialisme historique sur la production de cette époque, notamment celle du Karl Kautsky d'avant-guerre, en se présentant comme les véritables détenteurs de la légitimité de l'analyse marxiste de la Révolution française ${ }^{17}$. En 1932-1934, le débat qui oppose les sociaux-démocrates au sujet de la nature sociale et politique des décrets de Ventôse et plus généralement leur appréciation de l'œuvre du gouvernement révolutionnaire, en écho aux derniers travaux de l'historien français Albert Mathiez, offrent, à l'heure du triomphe du national-socialisme et de l'austro-fascisme, un exemple singulier du lien pouvant exister entre la politique contemporaine et l'usage des références révolutionnaires passées.

Au-delà des variations, des lectures diverses qu'elle suscite, la production socialedémocrate pendant ces quatre décennies constitue un moment historiographique qui permet de découvrir des approches et usages méconnus de l'histoire de la Révolution française. Au début du vingtième siècle, elle s'insère par exemple dans un ensemble d'études qui porte une attention inédite à l'histoire économique et sociale et à l'action des groupes populaires ; au côté de l'Histoire socialiste de Jean Jaurès et de La Grande Révolution de l'anarchiste russe Piotr Kropotkine, ce sont les premiers pas, «de l'Atlantique à l'Oural », d'une histoire économique et sociale des événements révolutionnaires, dont les prolongements traverseront des décennies d'historiographie. 
10 l'objet dans le cadre d'une organisation politique comme le SPD pose, dès la fin du dixneuvième siècle, le problème d'une articulation entre savoir scientifique et production militante qui, tout au long du vingtième siècle, aura des échos, notamment dans le cadre des partis communistes. Certaines spécificités comme le transfert des productions historiques de l'Allemagne vers l'Autriche (et aussi, dans une moindre mesure, les transferts inverses), les interactions avec d'autres espaces comme la Hongrie (en particulier les célébrations des premiers jacobins hongrois) - la liste n'est pas exhaustive - resteraient à étudier. Une histoire croisée de l'ensemble permettrait d'offrir un éclairage singulier sur l'historiographie ne se limitant pas à la seule étude de l'impact de la Révolution à l'étranger. Même pour des figures célèbres comme Jaurès ou Mathiez, l'usage des outils méthodologiques de l'histoire croisée permet de relire leurs visions supposées déjà connues et de comprendre combien la genèse de leurs travaux sur la Révolution française dépendait de lectures et de confrontations dépassant le seul Hexagone.

\section{NOTES}

1. Karl KAUTSKY, Die Klassengegensätze von 1789. Zum 100jährigen Gedenktag der grossen Revolution, Stuttgart: Dietz, 1889. En français : K. Kautsky, La lutte des classes en France en 1789, Paris : G. Jacques, 1901 (nouvelle traduction et édition: Karl Kautsky, Jean Jaurès, Le socialisme et la Révolution française, Paris : Demopolis, 2010

2. Voir par exemple Claude MAZAURIC, L'histoire de la Révolution française et la pensée marxiste, Paris : PUF, 2009. Voir aussi les souvenirs de l'historien Walter MARKOV, Wie viele Leben lebt der Mensch, Leipzig : Faber, 2009.

3. Sur l'espace germanophone voir Paul PASTEUR, Pratiques politiques et militantes de la socialdémocratie autrichienne 1888-1934, Paris : Belin, 2003.

4. Sans exclusive, voir par exemple Emmanuel JOUSSE, Réviser le marxisme? D’Édouard Bernstein à Albert Thomas, 1894-1914, Paris: L'Harmattan, 2007; Amaury CATEL, Le traducteur et le démiurge. Hermann Ewerbeck, un communiste allemand à Paris (1841-1860), mémoire de master d'histoire sous la direction de Christophe Prochasson, EHESS, 2009.

5. Georges HAUPT, L'historien et le mouvement social, Paris : Maspero, 1980.

6. Les études sur les transferts ont été nombreuses depuis l'article : Michael WERNER et Michel ESPAGNE, «La construction d'une référence allemande en France. Genèse et histoire culturelle» in: Annales ESC, vol. 42, $\mathrm{n}^{\circ}$ 4, 1987, p. 969-992. On peut citer par exemple un numéro récent d'Austriaca où figurent plusieurs contributions sur l'histoire de la gauche autrichienne: Ute wEINMANN (dir.), Autriche/France. Transferts d'idéesHistoires parallèles Sciences-Philosophie-Droit-Politique ( = Austriaca, vol. 63, 2006).

7. Michael WERNER et Bénédicte ZIMMERMANN, «Penser l'histoire croisée: entre empirie et réflexivité » in : Annales. Histoire, sciences sociales, vol. 58, n 1, 2003, p. 7-34. 
8. Alexandre ESCUDIER, «Épistémologies croisées ? L'impossible lecture des théoriciens allemands de l'histoire en France autour de $1900 »$ in : Michael WERNER et Bénédicte ZIMMERMANN (dir.), De la comparaison à l'histoire croisée, Paris : Seuil (Le genre humain), 2004, p. 139-177.

9. Heinrich CuNOW, Die revolutionäre Zeitungsliteratur Frankreichs während der Jahre 1789 bis 1794. Ein Beitrag zur Geschichte der französischen Klassen- und Parteikämpfe gegen Ende des 18. Jahrhunderts, Berlin: Buchhandlung Vorwärts, 1908 (réédité en 1912 sous le titre Die Parteien der Grossen Französischen Revolution und ihre Presse, Berlin : Buchhandlung Vorwärts Paul Singer, 1912).

10. Hermann WENDEL, Danton, Berlin : Rowohlt, 1930 (rééd. 1978).

11. Nous renvoyons le lecteur à la bibliographie de notre thèse : p. 509-554.

12. Notamment P. PASTEUR, Pratiques politiques..., op. cit. et Andrew BONnELL, The People's Stage in Imperial Germany: Social Democracy and Culture 1890-1914, Londres: Tauris Academic Studies, 2005.

13. Robert STUART, Marxism at Work. Ideology, class and french Socialism during the Third Republic, Cambridge : Cambridge University Press, 1992 ; Serge wolikow et Jean VIGREux (dir.), Cultures communistes au vingtième siècle : entre guerre et modernité, Paris : La Dispute, 2003 ; Eric HOBSBAWM et Terence RANGER, L'invention de la tradition (1983), Paris : Éditions Amsterdam, 2006.

14. La plupart de ces documents sont désormais référencés sur les sites internet de ces centres d'archives : voir www.iisg.nl et www.fes.de.

15. De ce point de vue, nous nous situons dans le prolongement du travail de Tamara KONDRATIEVA, Bolcheviks et Jacobins : itinéraire des analogies, Paris : Payot, 1989.

16. Emma ADLER, Die berühmten Frauen der französischen Revolution: 1789-1795, Wien : C.W. Stern, 1906.

17. Hermann DUNCKER (et alii), «Die grosse französische Revolution (1789-1799)» in: Marxistische Arbeiterschule, $\mathrm{n}^{\circ} 1$, Berlin, 1930.

\section{AUTEUR}

\section{JEAN-NUMA DUCANGE}

Jean-Numa Ducange est depuis le 1er septembre 2010 maître de conférences en histoire contemporaine à l'Université de Rouen 